\title{
Pose Machines: Articulated Pose Estimation via Inference Machines
}

\author{
Varun Ramakrishna, Daniel Munoz, Martial Hebert, \\ J. Andrew Bagnell, and Yaser Sheikh
}

The Robotics Institute, Carnegie Mellon University

\begin{abstract}
State-of-the-art approaches for articulated human pose estimation are rooted in parts-based graphical models. These models are often restricted to tree-structured representations and simple parametric potentials in order to enable tractable inference. However, these simple dependencies fail to capture all the interactions between body parts. While models with more complex interactions can be defined, learning the parameters of these models remains challenging with intractable or approximate inference. In this paper, instead of performing inference on a learned graphical model, we build upon the inference machine framework and present a method for articulated human pose estimation. Our approach incorporates rich spatial interactions among multiple parts and information across parts of different scales. Additionally, the modular framework of our approach enables both ease of implementation without specialized optimization solvers, and efficient inference. We analyze our approach on two challenging datasets with large pose variation and outperform the state-of-the-art on these benchmarks.
\end{abstract}

\section{Introduction}

There are two primary sources of complexity in estimating the articulated pose of a human from an image. The first arises from the large number of degrees of freedom (nearly 20) of the underlying articulated skeleton which leads to a high dimensional configuration space to search over. The second is due to the large variation in appearance of people in images. The appearance of each part can vary with configuration, imaging conditions, and from person to person.

To deal with this complexity, current approaches $[1,2,3,4,5,6]$ adopt a graphical model to capture the correlations and dependencies between the locations of the parts. However, inference in graphical models is difficult and inexact in all but the most simple models, such as a tree-structured or star-structured model. These simplified models are unable to capture important dependencies between locations of each of the parts and lead to characteristic errors. One such errordouble counting (see Figure 1) - occurs when the same region of the image is used to explain more than one part. This error occurs because of the symmetric appearance of body parts (e.g., the left and right arm usually have similar appearance) and that it is a valid configuration for parts to occlude each other. Modeling this appearance symmetry and self-occlusion with a graphical model 


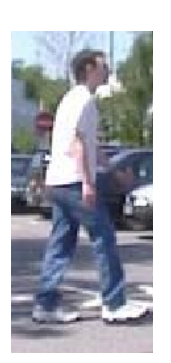

Input Image
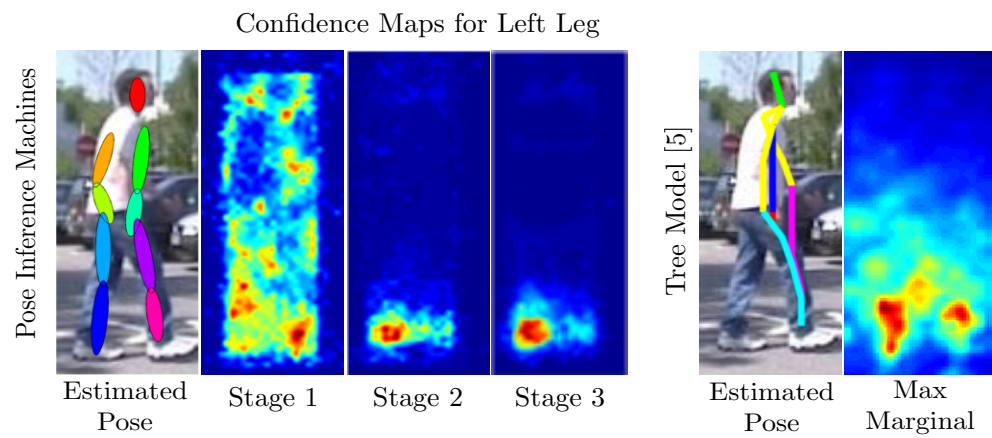

Fig. 1: Reducing double counting errors. By modelling richer interactions we prevent the double counting errors that occur in tree models. On the left we show the belief for the left foot of the person in each stage from our method. The belief quickly converges to a single sharp peak. On the right, we see that the tree-structured model [5] has a max-marginal for the left foot with multiple peaks and resulting in both legs being placed on the same area in the image.

requires additional edges and induces loops in the graph. Such non-tree structured graphical models typically require the use of approximate inference (e.g., loopy belief propagation), which makes parameter learning difficult [7].

A second limitation of graphical models is that defining the potential functions requires careful consideration when specifying the types of interactions. This choice is usually dominated by parametric forms such as simple quadratic models in order to enable tractable inference [1]. Finally, to further enable efficient inference in practice, many approaches are also restricted to use simple classifiers such as mixtures of linear models for part detection [5]. These are choices guided by tractabilty of inference rather than the complexity of the data. Such trade-offs result in a restrictive model that do not address the inherent complexity of the problem.

Our approach avoids this complexity vs. tractability trade-off by directly training the inference procedure. We present a method for articulated human pose estimation that builds off the hierarchical inference machine originally used for scene parsing $[8,9]$. Conceptually, the presented method, which we refer to as a Pose Machine, is a sequential prediction algorithm that emulates the mechanics of message passing to predict a confidence for each variable (part), iteratively improving its estimates in each stage. The inference machine architecture is particularly suited to tackle the main challenges in pose estimation. First, it incorporates richer interactions among multiple variables at a time, reducing errors such as double counting, as illustrated in Figure 1. Second, it learns an expressive spatial model directly from the data without the need for specifying the parametric form of the potential functions. Third, its modular architecture allows the use of high capacity predictors which are better suited to deal with the highly multi-modal appearance of each part. Inspired by recent work $[10,11]$ that has demonstrated the importance of conditioning finer part detection on 
the detection of larger composite parts in order to improve localization, we incorporate these multi-scale cues in our framework by also modeling a hierarchy of parts.

Our contributions include a method that simultaneously addresses the two said primary challenges of articulated pose estimation using the architecture of an inference machine. Additionally, our approach is simple to implement, requiring no specialized optimization solvers at test time, and is efficient in practice. Our analysis on two challenging datasets demonstrates that our approach improves upon the state-of-the-art and offers an effective, alternative framework to address the articulated human pose estimation problem.

\section{Related Work}

There is a vast body of work on the estimation of articulated human pose from images and video. We focus on methods to estimate the 2D pose from a single image. The most popular approach to pose estimation from images has been the use of pictorial structures. Pictorial structure models [1,2,3,4,5,6], express the human body as a tree-structured graphical model with kinematic priors that couple connected limbs. These methods have been successful on images where all the limbs of the person are visible, but are prone to characteristic errors such as double-counting image evidence, which occur because of correlations between variables that are not modeled by a tree-structured model.

Pictorial structure models with non-tree interactions have been employed $[12,13,14,15]$ to estimate pose in a single image. These models augment the tree-structure to capture occlusion relationships between parts not linked in the tree. Performing exact inference on these models is typically intractable and approximate methods at learning and test time need to be used. Recent methods have also explored using part hierarchies [16,17] and condition the detection of smaller parts that model regions around anatomical joints on the localization of larger composite parts or poselets $[11,10,18,19]$ that model limbs in canonical configurations and tend to be easier to detect.

The above models usually involve some degree of careful modeling. For example, [3] models deformation priors by assuming a parametric form for the pairwise potentials, and [5] restricts the appearance of each part to belong to a mixture model. These trade-offs are usually required to enable tractable learning and inference. Even so, learning the parameters of these models usually involves fine-tuned solvers or approximate piecewise methods. Our method does not require a tailor-made solver, as its modular architecture allows us to leverage well-studied algorithms for the training of supervised classifiers.

In [20], the authors use a strong appearance model, by training rotation dependent part detectors with separate part detectors for the head and torso while using a simple tree-structured model. In [21] better part detectors are learned by using multiple stages of random forests. However this approach uses a tree-structured graphical model to enforce spatial consistency. Our approach generalizes the notion of using the output of a previous stage to improve part 
localization, learns a spatial model in a non-parametric data-driven fashion and does not require the design of part-specific classifiers.

Our method bears some similarity to deep learning methods [22] in a broad sense of also being a multi-layered modular network. However, in contrast to deep-learning methods which are trained in a global fashion (e.g., using backpropagation), each module is trained locally in a supervised manner.

Our method reduces part localization to a sequence of predictions. The use of sequential predictions - feeding the output of predictors from a previous stage to the next - has been revisited in the literature from time to time. Methods such as $[23,24]$ applied sequential prediction to natural language processing tasks. While [25] explored the use of context from neighboring pixel classifiers for computer vision tasks. Our approach is based on the hierarchical inference machine architecture $[8,9]$ that reduces structured prediction tasks to a sequence of simple machine learning subproblems. Inference machines have been previously studied in image and point cloud labeling applications $[8,26]$. In this work, our contribution is to extend and analyze the inference machine framework for the task of articulated pose estimation.

\section{Pose Inference Machines}

\subsection{Background}

We view the articulated pose estimation problem as a structured prediction problem. That is, we model the pixel location of each anatomical landmark (which we refer to as a part) in the image, $Y_{p} \in \mathcal{Z} \subset \mathbb{R}^{2}$, where $\mathcal{Z}$ is the set of all $(u, v)$ locations in an image. Our goal is to predict the structured output $Y=\left(Y_{1}, \ldots, Y_{P}\right)$ for all $P$ parts. An inference machine consists of a sequence of multi-class classifiers, $g_{t}(\cdot)$, that are trained to predict the location of each part. In each stage $t \in\{1 \ldots T\}$, the classifier predicts a confidence for assigning a location to each part $Y_{p}=z, \forall z \in \mathcal{Z}$, based on features of the image data $\mathbf{x}_{z} \in \mathbb{R}^{d}$ and contextual information from the preceeding classifier in the neighborhood around each $Y_{p}$. In each stage, the computed confidences provide an increasingly refined estimate for the variable. For each stage $t$ of the sequence, the confidence for the assignment $Y_{p}=z$ is computed and denoted by

$$
b_{t}\left(Y_{p}=z\right)=g_{t}^{p}\left(\mathbf{x}_{z} ; \bigoplus_{i=1}^{P} \psi\left(z, \mathbf{b}_{t-1}^{i}\right)\right) \text {, }
$$

where

$$
\mathbf{b}_{t-1}^{p}=\left\{b_{t-1}\left(Y_{p}=z\right)\right\}_{z \in \mathcal{Z}},
$$

is the set of confidences from the previous classifier evaluated at every location $z$ for the $p$ 'th part. The feature function $\psi: \mathcal{Z} \times \mathbb{R}^{|\mathcal{Z}|} \rightarrow \mathbb{R}^{d_{c}}$ computes contextual features from the classifiers' previous confidences, and $\bigoplus$ denotes an operator for vector concatenation. 


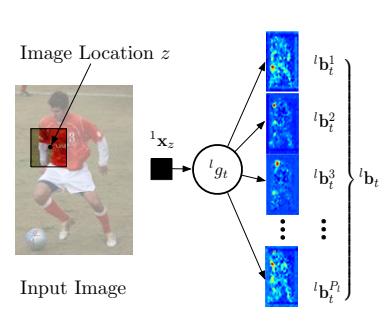

(a)

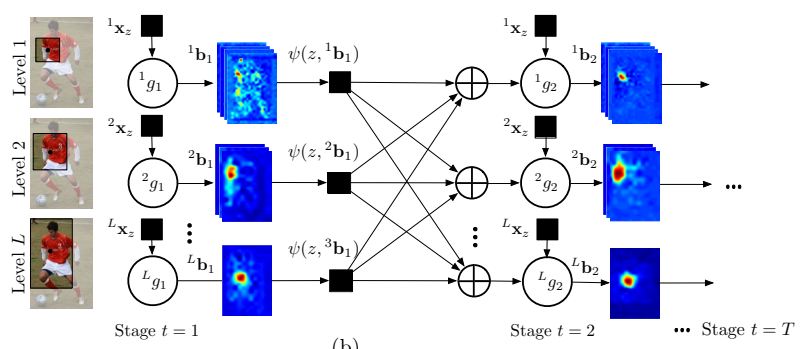

(b)

Fig. 2: (a) Multi-class prediction. A single multiclass predictor is trained for each level of the hierarchy to predict each image patch into one of $P_{l}+1$ classes. By evaluating each patch in the image, we create a set of confidence maps ${ }^{l} \mathbf{b}_{t}$. (b) Two stages of a pose inference machine. In each stage, a predictor is trained to predict the confidence of the output variables. The figure depicts the message passing in an inference machine at test time. In the first stage, the predictors produce an estimate for the confidence of each part location based on features computed on the image patch. Predictors in subsequent stages, refine these confidences using additional information from the outputs of the previous stage via the context feature function $\psi$.

Unlike traditional graphical models, such as pictorial structures, the inference machine framework does not need explicit modeling of the dependencies between variables via potential functions. Instead, the dependencies are arbitrarily combined using the classifier, which potentially enables complex interactions among the variables. Directly training the inference procedure via a sequence of simpler subproblems, allows us to use any supervised learning algorithm to solve each subproblem. We are able to leverage the state-of-the-art in supervised learning and use a sophisticated predictor capable of handling multi-modal variation. As detailed in the following section, our approach to articulated pose estimation takes the form of a hierarchical mean-field inference machine [8], where the contextual information that each variable uses comes from neighboring variables in both scale and space in the image.

\subsection{Incorporating a Hierarchy}

Recent work $[11,10]$ has shown that part detections conditioned on the location of larger composite parts improves pose estimation performance; however, these composite parts are often constructed to form tree graph structures [16]. Inspired by these recent advances, we design a hierarchical inference machine that similarly encodes these interactions among parts at different scales in the image. We define a hierarchy of parts from smaller atomic parts to larger composite parts. Each of the $L$ levels of the hierarchy have parts of a different type. At the coarsest level, the hierarchy is comprised of a single part that captures the whole body. The next level of the hierarchy is comprised of composite parts that model full limbs, while the finest level of the hierarchy is comprised of small parts that model a region around an anatomical landmark. We denote by $P_{1}, \ldots, P_{L}$, the 
number of parts in each of the $L$ levels of the hierarchy. In the following, we denote ${ }^{l} g_{t}^{p}(\cdot)$ as the classifier in the $t^{\text {th }}$ stage and $l^{\text {th }}$ level that predicts the score for the $p^{\text {th }}$ part. While separate predictors could be trained for each part $p$ in each level $l$ of the hierarchy, in practice, we use a single multi-class predictor that produces a set of confidences for all the parts from a given feature vector at a particular level in the hierarchy. For simplicity, we drop the superscript and denote this multi-class classifier as ${ }^{l} g_{t}(\cdot)$.

To obtain an initial estimate of the confidences for the location of each part, in the first stage $(t=1)$ of the sequence, a predictor ${ }^{l} g_{1}(\cdot)$ takes as input features computed on a patch extracted at an image location $z$, and classifies the patch into one of $P_{l}$ part classes or a background class (see Figure 2a), for the parts in the $l^{\text {th }}$ level of the hierarchy. We denote by $\mathbf{x}_{z}^{l}$, the feature vector of an image patch for the $l^{\text {th }}$ level of the hierarchy centered at location $z$ in the image. A classifier for the $l^{\text {th }}$ level of the hierarchy in the first stage $t=1$, therefore produces the following confidence values:

$$
{ }^{l} g_{1}\left(\mathbf{x}_{z}^{l}\right) \rightarrow\left\{{ }^{l} b_{1}^{p}\left(Y_{p}=z\right)\right\}_{p \in 0 \ldots P_{l}},
$$

where ${ }^{l} b_{1}^{p}\left(Y_{p}=z\right)$ is the score predicted by the classifier ${ }^{l} g_{1}$ for assigning the $p^{\text {th }}$ part in the $l^{\text {th }}$ level of the hierarchy in the first stage at image location $z$. Analogous to Equation 2, we represent all the confidences of part $p$ of level $l$ evaluated at every location $z=(u, v)^{T}$ in the image as ${ }^{l} \mathbf{b}_{t}^{p} \in \mathbb{R}^{w \times h}$, where $w$ and $h$ are the width and height of the image, respectively. That is,

$$
{ }^{l} \mathbf{b}_{t}^{p}[u, v]={ }^{l} b_{t}^{p}\left(Y_{p}=(u, v)^{T}\right) .
$$

For convenience, we denote the collection of confidence maps for all the parts belonging to level $l$ as ${ }^{l} \mathbf{b}_{t} \in \mathbb{R}^{w \times h \times P_{l}}$ (see Figure 2a).

In subsequent stages, the confidence for each variable is computed similarly to Equation 1. In the order to leverage the context across scales/levels in the hierarchy, the prediction is defined as

$$
{ }^{l} g_{t}\left(\mathbf{x}_{z}^{l}, \bigoplus_{l \in 1 \ldots L} \psi\left(z,{ }^{l} \mathbf{b}_{t-1}\right)\right) \rightarrow\left\{{ }^{l} b_{t}^{p}\left(Y_{p}=z\right)\right\}_{p \in 0 \ldots P_{l}}
$$

As shown in Figure 2b, in the second stage, the classifier ${ }^{l} g_{2}$ takes as input the features $\mathbf{x}_{z}^{l}$ and features computed on the confidences via the feature function $\psi$ for each of the parts in the previous stage. Note that the the predictions for a part use features computed on outputs of all parts and in all levels of the hierarchy $\left(\left\{{ }^{l} \mathbf{b}_{t-1}\right\}_{l \in 1 \ldots L}\right)$. The inference machine architecture allows learning potentially complex interactions among the variables, by simply supplying features on the outputs of the previous stage (as opposed to specifying potential functions in a graphical model) and allowing the classifier to freely combine contextual information by picking the most predictive features. The use of outputs from all neighboring variables, resembles the message passing mechanics in variational mean field inference [9]. 


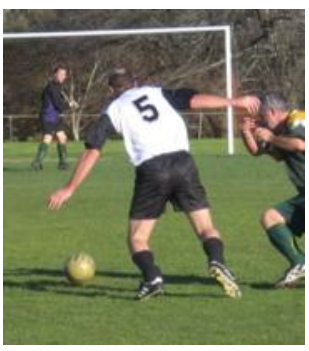

Input Image (a)

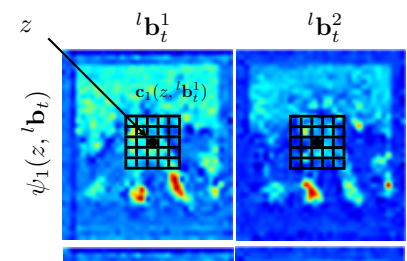

(b)

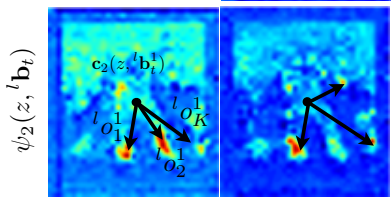

${ }^{l} \mathbf{b}_{t}^{3}$
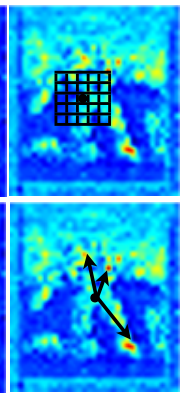

${ }^{l} \mathbf{b}_{t}^{P_{l}}$
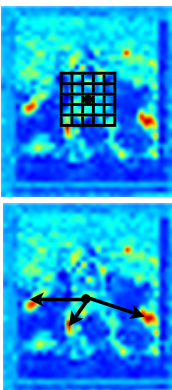

Fig. 3: Context Feature Maps (a) Context patch features are computed from each score map for each location. The figure illustrates a $5 \times 5$ sized context patch (b) The context offset feature comprises of offsets to a sorted list of peaks in each score map.

\subsection{Context Features}

To capture the spatial correlations between the confidences of each part with respect to its neighbors, we describe two types of factors with associated "context" feature maps denoted by $\psi_{1}$ and $\psi_{2}$.

Context Patch Features. The feature map $\psi_{1}$ at a location $z$ takes as input the confidence maps for the location of each part in a hierarchy level $l$ and produces a feature that is a vectorized patch of a predefined width extracted at the location $z$ in the confidence map ${ }^{l} \mathbf{b}_{t}^{p}$ (see Figure 3a). We denote the set of patches extracted and vectorized at the location $z$, from the beliefs of the parts in the hierarchy level $l$, by $\mathbf{c}_{1}\left(z,{ }^{l} \mathbf{b}_{t-1}^{p}\right)$. The feature map $\psi_{1}$ is therefore given by:

$$
\psi_{1}\left(z,{ }^{l} \mathbf{b}_{t-1}\right)=\bigoplus_{p \in 0 \ldots P_{l}} \mathbf{c}_{1}\left(z,{ }^{l} \mathbf{b}_{t-1}^{p}\right)
$$

In words, the context feature is a concatenation of scores at location $z$ extracted from the confidence maps of all the parts in each level the hierarchy. The context patch encodes neighboring information around location $z$ as would be passed as messages in a factor graph. Note that because we encode the context from all parts, this would be analogous to having a graphical model with a complete graph structure and would be intractable to optimize.

Context Offset Features. We compute a second type of feature, $\psi_{2}$, in order to encode long-range interactions among the parts that may be at non-uniform, relative offsets. First, we perform non-maxima suppresion to obtain a sorted list of $K$ peaks from each of the $P_{l}$ confidence maps ${ }^{l} \mathbf{b}_{t-1}^{p}$ for all the parts in the $l^{\prime}$ th hierarchy level. Then, we compute the offset vector in polar coordinates from location $z$ to each $k^{\text {th }}$ peak in the confidence map of the $p^{\text {th }}$ part and $l^{\text {th }}$ level denoted as as ${ }^{l} o_{k}^{p} \in \mathbb{R}^{+} \times \mathbb{R}$ (see Figure $3 \mathrm{~b}$ ). The set of context offset features computed from one part's confidence map is defined as:

$$
\mathbf{c}_{2}\left(z,{ }^{l} \mathbf{b}_{t-1}^{p}\right)=\left[{ }^{l} o_{1}^{p} ; \ldots ;{ }^{l} o_{K}^{p}\right] .
$$




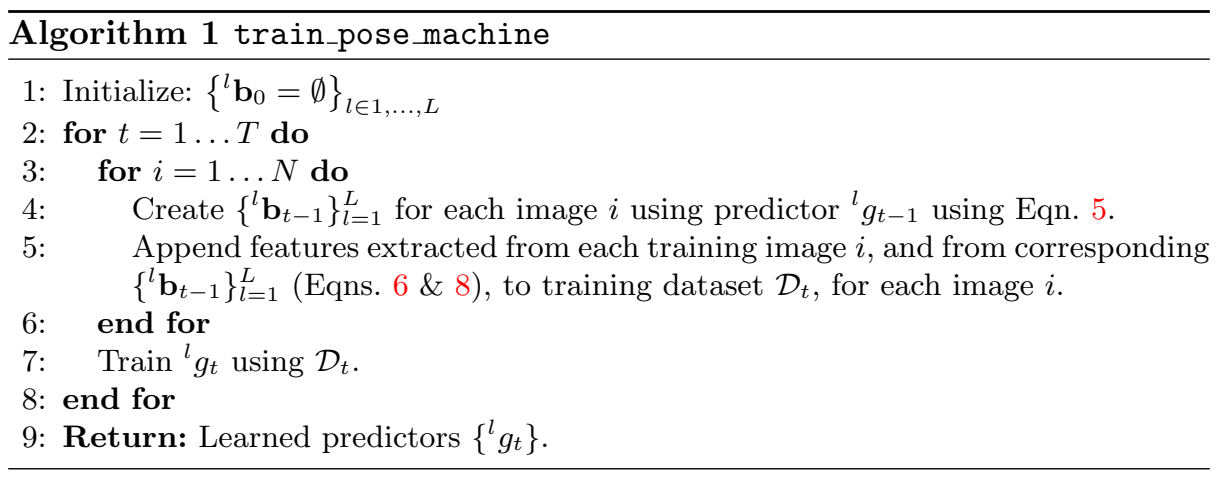

Then, the context offset feature map $\psi_{2}$ is formed by concatenating the context offset features $\mathbf{c}_{2}\left(z,{ }^{l} \mathbf{b}_{t-1}^{p}\right)$ for each part in the the hierarchy:

$$
\psi_{2}\left(z,{ }^{l} \mathbf{b}_{t-1}\right)=\bigoplus_{p \in 1 \ldots P_{l}} \mathbf{c}_{2}\left(z,{ }^{l} \mathbf{b}_{t-1}^{p}\right) .
$$

The context patch features $\left(\psi_{1}\right)$ capture coarse information regarding the confidence of the neighboring parts while the offset features $\left(\psi_{2}\right)$ capture precise relative location information. The final context feature $\psi$ is computed by concatenating two: $\psi(\cdot)=\left[\psi_{1}(\cdot) ; \psi_{2}(\cdot)\right]$.

\subsection{Training}

Training the inference procedure involves directly training each of the predictors, $\left\{{ }^{l} g_{t}\right\}$, in each level $l \in\{1, \ldots, L\}$, and for each stage $t \in\{1, \ldots, T\}$. We describe our training procedure in Algorithm 1. Training proceeds in a stage-wise manner. The first set of predictors $\left\{{ }^{l} g_{1}\right\}$ are trained using a dataset $\mathcal{D}_{0}$ consisting of image features on patches extracted from the training set of images at the annotated landmarks. For deeper stages, the dataset $\mathcal{D}_{t}$ is created by extracting and concatenating the context features from the confidence maps $\left\{{ }^{l} \mathbf{b}_{t-1}\right\}_{l=1}^{L}$ for each image, at the annotated locations.

\subsection{Stacking}

Training the predictors of such an inference procedure is prone to overfitting. Using the same training data to train the predictors in subsequent stages will cause them to rely on overly optimistic context from the previous stage, or overfit to idiosyncrasies of that particular dataset. Ideally we would like to train the subsequent stages with the output of the previous stages similar to that encountered at test time. In order to achieve this, we use the idea of stacked training $[27,23]$.

Stacked training aims to prevent predictors trained on the output of the first stage from being trained on same training data. Stacking proceeds similarly to 


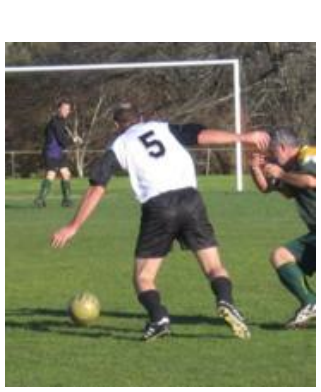

Input Image
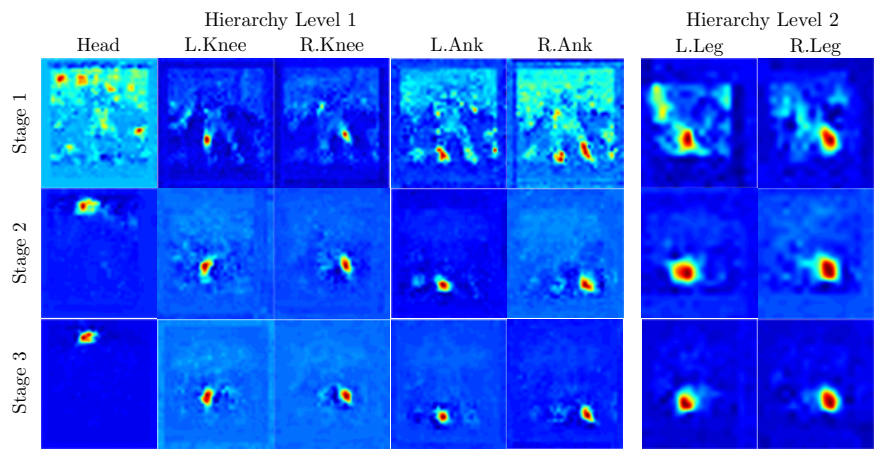

Fig. 4: The output of a three stage pose inference machine at each stage. An inference machine iteratively produces more refined estimates of the confidence for the location of each part. In the first stage, the estimate produced only from image features is noisy and has multiple modes. Subsequent stages refine the confidence based on predictions from neighboring factors to a sharp unimodal response at the correct location and suppress false positive responses in the background. The confidences from left to right are for the head, left-knee, right-knee, left-ankle, right-ankle, left-leg, rightleg.

cross-validation by making $M$ splits of the training data $\mathcal{D}$ into training and held-out data $\left\{\mathcal{D}^{m}, \mathcal{D} / \mathcal{D}^{m}\right\}_{m=1 \ldots M}$. For each predictor we aim to train in the first stage, we make $M$ copies, each trained on one of the $M$ splits of the training data. To create the training data for the next stage, for each training sample, we use the copy of the predictor that has not seen the sample (i.e., the sample is in the held-out data for that predictor). Proceeding in this way creates a dataset to train the next stage on the outputs of the previous stage, ensuring that the outputs mimic test-time behavior. We repeat the stacking procedure for each subsequent stage. The stacking procedure is only performed during training to create a training dataset for subsequent stages. At test time, we use a predictor in each stage that is trained using all of the data.

\subsection{Inference}

At test time, inference proceeds in a sequential fashion as show in Figure 2b. Features are extracted from patches of different scales (corresponding to each of the $L$ levels of the hierarchy) at each location in the image and input to the first stage classifiers $\left\{{ }^{l} g_{1}\right\}_{l=1}^{L}$, resulting in the output confidence maps $\left\{{ }^{l} \mathbf{b}_{1}\right\}_{l=1}^{L}$. Messages are passed to the classifiers in the next stage, by computing context features via the feature maps $\psi_{1}, \psi_{2}$ on the confidences ${ }^{l} \mathbf{b}_{1}$ from the previous stage. Updated confidences $\left\{{ }^{l} \mathbf{b}_{2}\right\}_{l=1}^{L}$ are computed by the classifiers ${ }^{l} g_{2}$ and this procedure is repeated for each stage. The computed confidences are increasingly refined estimates for the location of the part as shown in Figure 4. The location of each part is then computed as,

$$
\forall l, \forall p,{ }^{l} y_{p}^{*}=\underset{z}{\operatorname{argmax}}{ }^{l} \mathbf{b}_{T}^{p}(z) .
$$


The final pose is computed by directly picking the maxima of the confidence map of each part after the final stage.

\subsection{Implementation}

Choice of Predictor. The modular nature of the inference machine architecture allows us to insert any supervised learning classifier as our choice of multi-class predictor $g$. As the data distribution is highly multi-modal, a high-capacity nonlinear predictor is required. In this work, we use a boosted classifier [28] with random forests for the weak learners, because random forests have been empirically shown to consistently outperform other methods on several datasets [29]. We learn our boosted classifier by optimizing the non-smooth hinge loss [30]. We use 25 iterations of boosting, with a random forest classifier. Each random forest classifier consists of 10 trees, with a maximum depth of 15 and with a split performed only if a node contained greater than 10 training samples.

Training. To create positive samples for training, we extract patches around the annotated anatomical landmarks in each training sample. For the background class, we use patches sampled from a negative training corpus as in [5]. In addition, in subsequent stages, we sample negative patches from false positive regions in the positive images.

Image Features. We extract a set of image features from a patch at each location in the image. We use a standard set of simple features to provide a direct comparison and to control for the effect of features on performance. We use Histogram of Gradients (HOG) features, Lab color features, and gradient magnitude. The HOG features are defined based on the structure of the human poses labeled in the respective datasets, which we detail in the follow section. In the FLIC dataset [11], only an upper-body model is annotated and we use 6 orientations with a bin size 4. In the LEEDS dataset [6], a full body model is annotated and we use 6 orientations with a bin size of 8 in the finest level of the hierarchy. We increase the bin size by a factor of two for the coarser levels in the hierarchy. For the upper body model, we model each part in the finest level of the hierarchy with $9 \times 9$ HOG cells, while we use $5 \times 5$ HOG cells for the full body model. These parameter choices are guided by previous work using these datasets $[11,5]$.

Context Features. For the context patch features, we use a context patch of size $21 \times 21$, with max-pooling in each $2 \times 2$ neighborhood resulting in a set of 121 numbers per confidence map. For the context offset features we use $K=3$ peaks.

\section{Evaluation}

We evaluate and compare the performance of our approach on two standard pose estimation datasets to the current state-of-the-art methods. 


\begin{tabular}{lccccccc}
\hline Method & Torso & $\begin{array}{c}\text { Upper } \\
\text { Legs }\end{array}$ & $\begin{array}{c}\text { Lower } \\
\text { Legs }\end{array}$ & $\begin{array}{c}\text { Upper } \\
\text { Arms }\end{array}$ & $\begin{array}{c}\text { Lower } \\
\text { Arms }\end{array}$ & Head & Total \\
\hline Ours & $\mathbf{9 3 . 1}$ & $\mathbf{8 3 . 6}$ & $\mathbf{7 6 . 8}$ & $\mathbf{6 8 . 1}$ & 42.2 & 85.4 & $\mathbf{7 2 . 0}$ \\
\hline Pishchulin [20] & 88.7 & 78.8 & 73.4 & 61.5 & $\mathbf{4 4 . 9}$ & $\mathbf{8 5 . 6}$ & 69.2 \\
Pishchulin [10] & 87.5 & 75.7 & 68.0 & 54.2 & 33.8 & 78.1 & 62.9 \\
Yang\&Ramanan [5] & 84.1 & 69.5 & 65.6 & 52.5 & 35.9 & 77.1 & 60.8 \\
Eichner\&Ferrari [31] & 86.2 & 74.3 & 69.3 & 56.5 & 37.4 & 80.1 & 64.3 \\
\hline
\end{tabular}

Table 1: Quantitative performance on LEEDS Sports Pose dataset. Performance is measured by the PCP metric on the test set of the LEEDS sports dataset. Our algorithm outperforms all current methods.

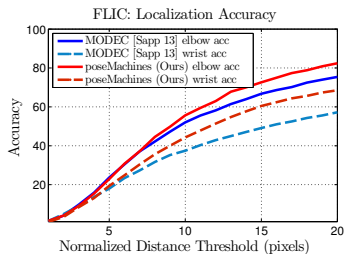

(a)

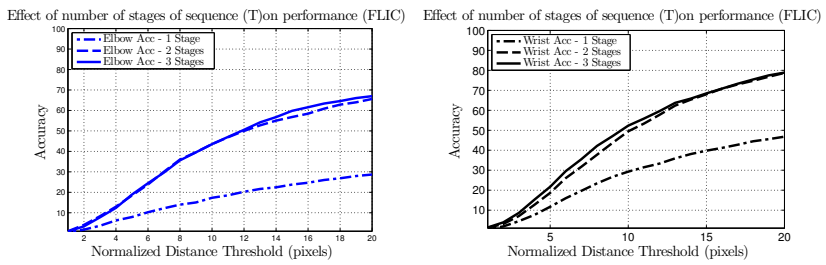

(b)

Fig. 5: (a) Comparison to state-of-the-art on FLIC Elbow and wrist localization accuracy on the FLIC dataset. We achieve higher accuracies for both joints compared to the state-of-the-art [11]. (b) Effect of number of stages. We plot the change in accuracy with the number of stages in the sequence. We observe that including a second stage which uses contextual information greatly increases the performance. We also observe a slight improvement with the incorporation of an additional third stage.

LEEDS Sports Pose Dataset. We evaluate our approach on the LEEDS sports dataset [6] which consists of 1,000 images for training and 1,000 images for testing. The images are of people in various sport poses. We use the observercentric annotations as used in [10] for training and testing. We train a full body model comprised of a 2-level hierarchy. The second level of the hierarchy comprises of the 14 parts corresponding to each of the annotated joints. The first level comprises of 6 composite parts formed by grouping parts belonging to each of the limbs, a composite part for the head and shoulders and a composite part for the torso. Parameter choices were guided by a grid search using a development subset of the training dataset comprising of 200 images. We use the Percentage Correct Parts (PCP) metric to evaluate and compare our performance on the dataset. The results are listed in the Table 1. We outperform existing methods and achieve an average PCP score of 72.0. We show qualitative results of our algorithm on a few representative samples from the LEEDS dataset in Figure 7.

FLIC Upper Body Pose Dataset. We also evaluate our approach on the FLIC dataset [11] which consists of still frames from movies. The dataset consists of 4,000 images for training and 1,000 images for testing. We use a model trained to recognize the pose of the upper body. We employ a two-level hierarchy, with 

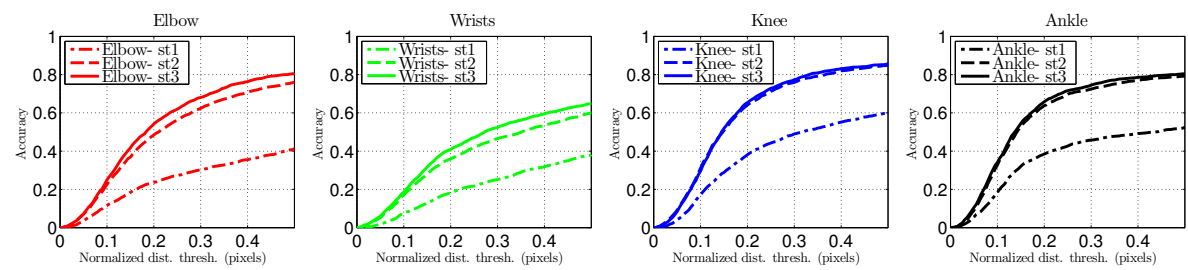

Fig. 6: Effect of number of stages on LSP. We plot the change in accuracy with the number of stages in the sequence for difficult landmarks on the LEEDS Sports dataset. The additional stages improve the performance especially of difficult parts like the elbows and wrists.

the finest level of the hierarchy comprising of seven parts corresponding to the annotated anatomical landmark locations, the second level comprising of three composite parts corresponding to each of the arms and one for the head and shoulders. Parameter choices were guided by a grid search using a development subset of the training dataset comprising of 200 images. We use the accuracy metric specified in [11]. In Figure 5 a we plot the accuracy of the wrist and elbow joints. Our approach shows a significant improvement over the state of the art [11]. We show qualitative results of our algorithm on samples from the FLIC dataset in Figure 8.

Effect of the number of stages. We study the effect of increasing the number of stages $T$ in the inference machine. Figure $5 \mathrm{~b}$ plots the part localization accuracy as a function of the distance from the ground truth label on the FLIC dataset. We see that predicting part location only based on image features ( $T=$ 1) results in poor performance. The addition of a second stage $(T=2)$ that incorporates contextual information results in a dramatic increase in the accuracy. An additional third stage $(T=3)$ adds a minor increase in performance on this dataset. Setting the number of stages is similar to how the number of iterations are set for message-passing algorithms such as belief propagation. For datasets of different sizes the number of stages can be set by evaluating the change in loss after each iteration.

We plot the change in accuracy with the number of stages in the sequence for difficult landmarks on the LEEDS Sports dataset (see Figure 6). We observe that including a second stage which uses contextual information greatly increases the performance. We also observe slight improvements for the knees and ankles, and a significant improvement for the wrists and elbows upon adding a third stage.

\section{Discussion}

We have presented an inference machine for articulated human pose estimation. The inference machine architecture allows us to learn a rich spatial model and incorporate high-capacity supervised predictors, resulting in substantially improved pose estimation performance. One of the main challenges that remain 


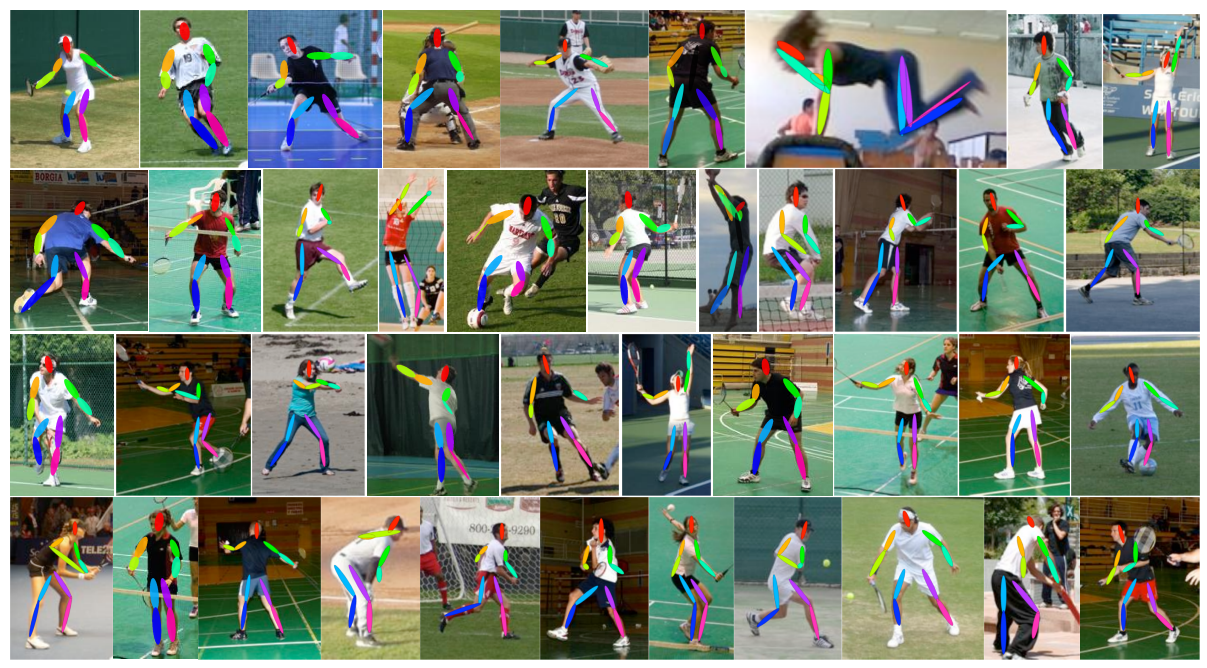

Fig. 7: Qualitative example results on the LEEDS sports dataset. Our algorithm is able to automatically learn a spatial model and correctly localize traditionally difficult parts such as the elbows and wrists.

is to correctly handle occluded poses, which is one of the failure modes of the algorithm (see Figure 9). A second failure mode is due to rare poses for which there are too few similar training instances. Tackling these challenges will need an understanding of the requirements from a human pose dataset for training an algorithm to work in the wild. The ability to handle complex variable dependencies leads to interesting directions for future work that include extending the method to monocular video by incorporating temporal cues, directly predicting poses in 3D, and adapting the method for different categories of articulated objects.

Acknowledgements: This material is based upon work supported by the National Science Foundation under Grants No. 1353120 and 1029679 and the NSF NRI Purposeful Prediction project.

\section{References}

1. Felzenszwalb, P.F., Huttenlocher, D.P.: Pictorial structures for object recognition. IJCV (2005)

2. Ramanan, D., Forsyth, D.A., Zisserman, A.: Strike a Pose: Tracking people by finding stylized poses. In: CVPR. (2005)

3. Andriluka, M., Roth, S., Schiele, B.: Monocular 3D Pose Estimation and Tracking by Detection. In: CVPR. (2010)

4. Andriluka, M., Roth, S., Schiele, B.: Pictorial Structures Revisited: People Detection and Articulated Pose Estimation. In: CVPR. (2009)

5. Yang, Y., Ramanan, D.: Articulated pose estimation with flexible mixtures-ofparts. In: CVPR. (2011) 


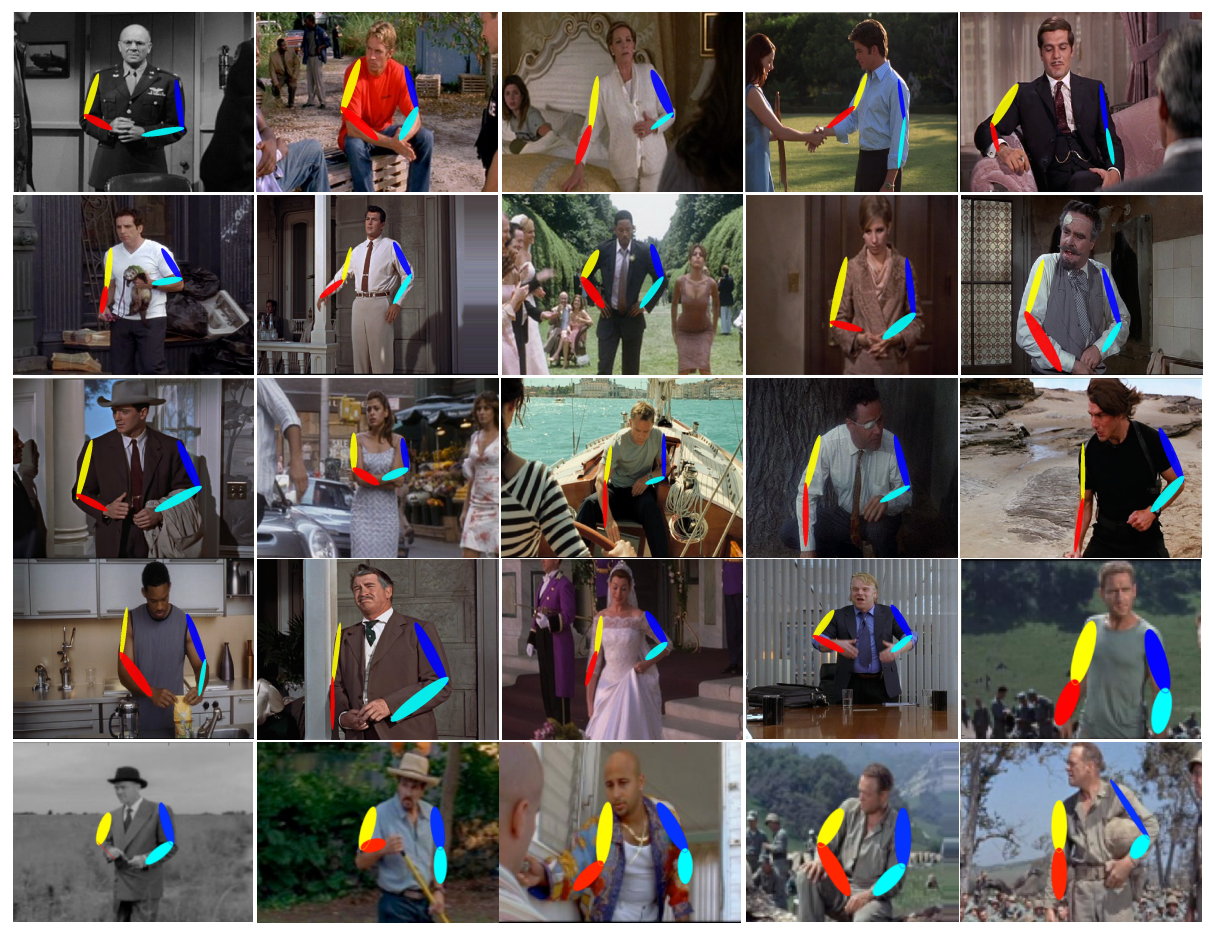

Fig. 8: Qualitative example results on the FLIC dataset. Our algorithm is able to automatically learn a spatial model and correctly localize traditionally difficult parts such as the elbows and wrists.
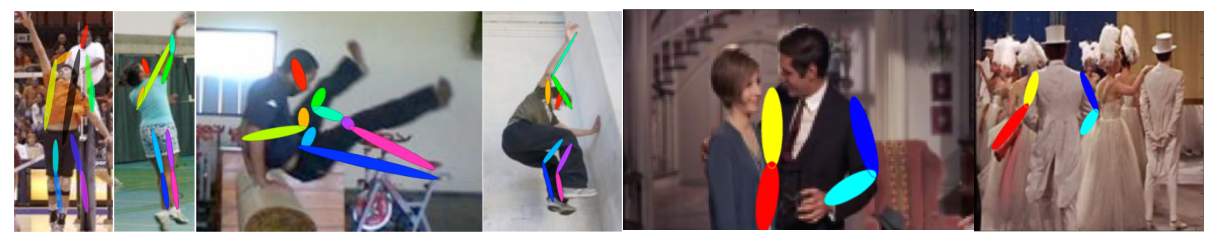

Fig. 9: Failure Modes. Typical failure modes include severe occlusion of parts and rare poses, for which too few training samples exist in the training set. The method is also prone to error when there are multiple people in close proximity.

6. Johnson, S., Everingham, M.: Clustered pose and nonlinear appearance models for human pose estimation. In: BMVC. (2010)

7. Kulesza, A., Pereira, F.: Structured learning with approximate inference. In: NIPS. (2007)

8. Munoz, D., Bagnell, J.A., Hebert, M.: Stacked hierarchical labeling. In: ECCV. (2010)

9. Ross, S., Munoz, D., Hebert, M., Bagnell, J.A.: Learning message-passing inference machines for structured prediction. In: CVPR. (2011) 
10. Pishchulin, L., Andriluka, M., Gehler, P., Schiele, B.: Poselet conditioned pictorial structures. In: CVPR. (2013)

11. Sapp, B., Taskar, B.: MODEC: Multimodal Decomposable Models for Human Pose Estimation. In: CVPR. (2013)

12. Wang, Y., Mori, G.: Multiple tree models for occlusion and spatial constraints in human pose estimation. In: ECCV. (2008)

13. Sigal, L., Black, M.J.: Measure locally, reason globally: Occlusion-sensitive articulated pose estimation. In: CVPR. (2006)

14. Lan, X., Huttenlocher, D.P.: Beyond trees: Common-factor models for $2 \mathrm{~d}$ human pose recovery. In: ICCV. (2005)

15. Karlinsky, L., Ullman, S.: Using linking features in learning non-parametric part models. In: ECCV. (2012)

16. Tian, Y., Zitnick, C.L., Narasimhan, S.G.: Exploring the spatial hierarchy of mixture models for human pose estimation. In: ECCV. Springer (2012)

17. Sun, M., Savarese, S.: Articulated part-based model for joint object detection and pose estimation. In: ICCV. (2011)

18. Gkioxari, G., Arbeláez, P., Bourdev, L., Malik, J.: Articulated pose estimation using discriminative armlet classifiers. In: CVPR, IEEE (2013)

19. Wang, Y., Tran, D., Liao, Z.: Learning hierarchical poselets for human parsing. In: CVPR, IEEE (2011)

20. Pishchulin, L., Andriluka, M., Gehler, P., Schiele, B.: Strong appearance and expressive spatial models for human pose estimation. In: ICCV. (2013)

21. Dantone, M., Gall, J., Leistner, C., Van Gool, L.: Human pose estimation using body parts dependent joint regressors. In: CVPR. (2013)

22. Bengio, Y.: Learning deep architectures for AI. Foundations and trends in Machine Learning (2009)

23. Carvalho, V., Cohen, W.: Stacked sequential learning. In: IJCAI. (2005)

24. Daumé III, H., Langford, J., Marcu, D.: Search-based structured prediction. Machine Learning (2009)

25. Bai, X., Tu, Z.: Auto-context and its application to high-level vision tasks and 3d brain image segmentation. PAMI (2009)

26. Xiong, X., Munoz, D., Bagnell, J.A., Hebert, M.: 3-d scene analysis via sequenced predictions over points and regions. In: ICRA. (2011)

27. Wolpert, D.H.: Stacked Generalization. Neural Networks (1992)

28. Friedman, J.H.: Greedy function approximation: a gradient boosting machine. Annals of Statistics (2001)

29. Caruana, R., Niculescu-Mizil, A.: An empirical comparison of supervised learning algorithms. In: ICML. (2006)

30. Grubb, A., Bagnell, J.A.: Generalized boosting algorithms for convex optimization. In: ICML. (2011)

31. Eichner, M., Ferrari, V.: Appearance sharing for collective human pose estimation. In: ACCV. (2012) 